\title{
Mindfulness em Ambientes Escolares: Adaptações e Protocolos Emergentes
}

\author{
Tiago Pires Tatton-Ramos ${ }^{1}$ \\ Reinaldo Augusto Gomes Simões \\ Fernando Lives Andela Niquice \\ Programa de Pós-Graduação em Psicologia da Universidade Federal do Rio Grande do Sul, \\ Porto Alegre, RS, Brasil \\ Lisiane Bizarro \\ Departamento de Psicologia do Desenvolvimento e da Personalidade da Universidade \\ Federal do Rio Grande do Sul, Porto Alegre, RS, Brasil \\ Tamara A. Russell \\ Institute of Psychiatry, Psychology and Neuroscience of King's College, London, UK
}

\section{Resumo}

Estudos têm demonstrado o impacto positivo das Mindfulness Based Interventions (MBIs) no tratamento de doenças e transtornos mentais em adultos. Recentemente, as MBIs estão sendo adaptadas para crianças e adolescentes em ambiente escolar. O objetivo deste artigo é discutir as adaptações necessárias, o papel da Autocompaixão, os efeitos das intervenções e o treinamento de professores através de três MBIs adaptadas para escolas: Dot-Be, MindUp e Learning to Breath. Os resultados indicam que MBIs para escolas devem ter menor duração das sessões; maior integração das práticas com o quotidiano; maior exploração dos cinco sentidos; maior utilização de metáforas, linguagem visual e tecnologia; e envolvimento de familiares, professores e educadores. Tanto o conceito quanto as práticas de Autocompaixão são utilizadas nestas MBIs, afetando a estrutura das intervenções e a escolha das práticas, e os efeitos nos desfechos pesquisados. Evidências sugerem que os efeitos do treinamento de Mindfulness em ambientes educacionais podem ser semelhantes àqueles verificados em adultos em contextos de saúde. Entretanto, com a proliferação de protocolos de treinamento para professores e gestores educacionais, as evidências de efetividade de MBIs neste contexto não podem ser generalizadas. Visando contribuir com esse processo, apresenta-se um modelo de treinamento em Mindfulness para a equipe educacional.

Palavras-chave: Mindfulness, autocompaixão, escolas, crianças, intervenção.

\section{Mindfulness in School Settings: Adjustments and Emerging Protocols}

\section{Abstract}

Several studies have demonstrated the positive impact of Mindfulness-Based Interventions (MBIs) in treatment of diseases and mental disorders in adults. MBIs are being adapted on children and

Endereço para correspondência: Universidade Federal do Rio Grande do Sul, Instituto de Psicologia, rua Ramiro Barcelos, Laboratório de Fenomenologia Experimental e Cognição, 2600, Porto Alegre, RS, Brasil 90035-003. Fones: (51) 9911-0542, (51) 3308-5261, (51) 3308-5246. Fax: (51) 3308-5473. E-mail: tiagotatton@gmail. com, fernandoniquice@gmail.com

Os autores agradecem à Coordenação de Aperfeiçoamento de Pessoal de Nível Superior (CAPES) pelo financiamento do trabalho. 
adolescents in school environment. This paper aims to discuss the necessary adaptations, the role of self-compassion, the interventions effects and the teacher training, through three MBIs adapted for schools: Dot-Be, MindUp, and Learning to Breath. The results indicate that MBIs for schools should be structured with shorter duration of sessions; greater integration of mindfulness exercises with daily activities; further exploration of the five senses; increased use of metaphors, visual language and technology; and the involvement of family members, teachers and educators. Both the concept and the practices of self-compassion are used in these MBIs, affecting the structure, the choice of practices and the effects on assessed outcomes. Evidence suggests that the effects of Mindfulness training in educational environments may be similar to those observed in adults in health contexts. However, with the proliferation of training protocols to teachers and educational managers, the evidence of MBIs effectiveness in the educational context should not be generalized. In order to contribute to this process, this paper presents a general training framework in Mindfulness for educational staff.

Keywords: Mindfulness, compassion, schools, children, intervention.

\section{Mindfulness en el Ambiente Escolar: Adaptaciones y Protocolos Emergentes}

\section{Resumen}

Estudios han demostrado el impacto positivo de Mindfulness Based Interventions (MBIs) en tratamiento de enfermedades y trastornos mentales en adultos. MBIs están siendo adaptadas para niños y adolescentes en ambiente escolar. El objetivo es analizar cambios necesarios, papel de la autocompasión, efectos de las intervenciones y formación del profesorado, a través de tres MBIs adaptados para escuelas: Dot-Be, MindUp y Learnig to Breath. Resultados indican que MBIs para escuelas deben tener sesiones de menor duración; mayor integración de las prácticas en la rutina diaria; mayor exploración de los cinco sentidos; mayor uso de metáforas, lenguaje visual y tecnología; y participación de la familia, profesores y personal educativo. Tanto el concepto y las prácticas de auto-compasión son utilizados en estos MBIs, afectando la estructura de intervenciones, la elección de práticas y los efectos sobre resultados. Evidencias sugieren que los efectos del entrenamiento en Mindfulness en entornos educativos pueden ser similares a los observados en adultos. Sin embargo, con la proliferación de protocolos de entrenamiento para maestros y administradores de la educación, evidencias de eficacia de los instrumentos no se pueden generalizar. Para contribuir a la optimización de las MBIs para escuelas, se presenta un modelo de formación para el personal educativo.

Palabras clave: Mindfulness, autocompasión, escuelas, niños, intervención.

Mindfulness é considerado um "fenômeno emergente" (Cullen, 2011) no universo acadêmico, particularmente nos campos da Psicologia e da Medicina Comportamental (Khoury et al., 2013; Langer, 1992; Ludwig \& Kabat-Zinn, 2008). Tal incremento evidencia-se no crescente número de publicações neste campo nas últimas três décadas e na utilização de métodos de pesquisa diversificados (Hölzel et al., 2011; Kabat-Zinn, 2003; Williams \& Kuyken, 2012). Estudos de revisão demonstram que altos escores em Mindfulness estão associados a desfechos físi- cos e mentais salutares para diversas condições (Chiesa \& Serretti, 2009; Eberth \& Sedlmeier, 2012; Grossman, Niemann, Schmidt, \& Walach, 2004; Khoury et al., 2013).

Somente nos últimos anos publicações em língua portuguesa começaram a explorar o tema, no Brasil (Barros, Kozasa, Souza, \& Ronzani, 2014; Hirayama et al., 2014; Menezes, Dell'Aglio, \& Bizarro, 2012; Silveira, Castro, \& Gomes, 2012; Tatton-Ramos, 2015; Vandenberghe \& Sousa, 2006) e também em Portugal (Duarte, Ferreira, \& Gouveia, 2010). No entan- 
to, estes estudos não abordam Mindfulness para crianças e jovens, tampouco a prática em ambientes escolares.

Brown e Ryan (2006) definem Mindfulness como um estado mental, ou disposição (traço), caracterizado por quatro características centrais: (a) não-elaborativo, (b) não-julgador, (c) centrado no aqui-e-agora e (d) onde pensamentos, sentimentos e sensações que surgem no campo atencional são reconhecidos e aceitos como tal. No estado de Mindfulness, o indivíduo procura observar diretamente suas experiências, ao invés de fazê-lo de modo automatizado, através de filtros de crenças e expectativas (Walsh, Balint, Smolira, Fredericksen, \& Madsen, 2009).

Além de um estado mental ou traço de personalidade, Mindfulness também pode ser entendido como uma prática, tal como se configura nas chamadas Mindfulness Based Interventions $^{2}$ (MBIs), intervenções que seguem protocolos. Neste caso, Mindfulness representa um exercício contínuo de um conjunto específico de práticas capazes de fomentar estes estados mentais (Brown \& Ryan, 2006). Atualmente, existem diversas MBIs, mas algumas estão sendo desenvolvidas e pesquisadas há décadas, como o Mindfulness Based Stress Reduction (MBSR; Kabat-Zinn, 1982) e o Mindfulness Based Cognitive Therapy (MBCT; Teasdale et al., 2000).

Universidades e instituições privadas ao redor do mundo oferecem treinamentos e certificações formais (Mindfulness Teacher Trainings), de curta e longa duração, para profissionais se capacitarem como instrutores de Mindfulness. Certificações deste tipo usualmente exigem a comprovação, pelo profissional interessado, da participação em algumas destas intervenções e, além disso, da manutenção de uma prática pessoal regular (Crane et al., 2013). Igualmente, existem regulamentações publicadas para avaliar a aderência e qualidade do ensino de Mindfulness por parte de instrutores (Crane et al., 2012).

2 Em português podemos chamar de Intervenções Baseadas em Mindfulness, mas aqui os autores preferiram utilizar o termo em inglês já consagrado na literatura da área.
Áreas como organizações e educação têm incluído, progressivamente, as MBIs em seus campos de intervenção e pesquisa (Cullen, 2011). Nestes contextos, a implementação de MBIs vem sendo realizada como estratégia de prevenção do surgimento de doenças e de baixa performance, e não como tratamento complementar de problemas físicos e psicológicos (Cullen, 2011). Um efeito direto destas mudanças é a expansão das MBIs na direção de populações mais jovens, como crianças e adolescentes (Frank, Jennings, \& Greenberg, 2013).

Nestas populações, o treinamento de competências de autorregulação emocional e comportamental tem sido considerado uma estratégia eficaz para a promoção de saúde e bem-estar generalizado e para a prevenção de sintomas de depressão e ansiedade (Meiklejohn et al., 2012; Roeser et al., 2013). Entretanto, a oferta, em larga escala, de MBIs para crianças e adolescentes exige o envolvimento da comunidade, especialmente de familiares, professores e gestores educacionais (Roeser et al., 2013).

Uma meta-análise realizada por Zenner, Herrnleben-Kurz, e Walach (2014) avaliou 13 estudos publicados, demonstrando que as MBIs são uma estratégia efetiva, de baixo custo, fácil aplicabilidade, que pode ser oferecida a estas populações em ambientes escolares. Embora a adaptação de MBIs para populações mais jovens esteja em curso há quase uma década, ainda é necessário esclarecer quais são: (a) as adaptações estruturais necessárias (duração, tipos de exercícios, linguagem, etc.) para aumentar a efetividade destas intervenções; (b) os protocolos emergentes que acumulam mais evidências de eficácia; (c) o papel da Autocompaixão enquanto componente central de efetividade; (d) os efeitos gerais das diferentes intervenções; (e) as estratégias de treinamento continuado para professores e equipe escolar que podem ser desenvolvidas (Burke, 2010; Carmody \& Baer, 2008; Joyce, Etty-Leal, Zazryn, \& Hamilton, 2010; Schonert-Reichl \& Lawlor, 2010). Este artigo está estruturado com o objetivo de apresentar, descrever e discutir estas cinco questões. Para tanto, três MBIs adaptadas para escolas, que acumulam ao menos três investigações empíricas, foram inclu- 
ídas: Dot-Be (.B), MindUp e Learning to Breath (L2b). Outras MBIs para escolas não entraram em nossa avaliação como, por exemplo, os programas Mindful Schools e Inner Kids. Estes programas estão sendo investigados empiricamente, mas os resultados publicados ainda são poucos. Por fim, o artigo pretende contribuir para a expansão e amadurecimento deste debate em língua portuguesa, especialmente no Brasil.

\section{A Questão da Autocompaixão en- quanto Componente Central da MBls}

A importância do treinamento em Autocompaixão vem crescendo ao longo dos anos na literatura acadêmica sobre Mindfulness e, da mesma forma, sua inserção nas MBIs (Neff, Rude, \& Kirkpatrick, 2007; Russell \& Tatton-Ramos, 2015; Woodruff et al., 2013). Nessa linha, existem atualmente alguns protocolos de Mindfulness baseados em Autocompaixão (Germer, 2009; Neff \& Germer, 2013).

Alguns estudiosos compreendem Mindfulness, sobremaneira, como um treinamento de atenção sustentada (Tang \& Posner, 2009). No entanto, nos exercícios de Mindfulness, a observação de distrações (mind wanderings) é parte do processo (Hayes \& Feldman, 2004). A progressiva curiosidade e a familiarização com reações automatizadas e inflexíveis da experiência do indivíduo são centrais ao exercício de Mindfulness (Hayes, Follette, \& Linehan, 2011). É neste sentido que Neff e Germer (2013) denominam a aceitação psicológica como Autocompaixão.

Nas MBIs que vêm sendo adaptadas para o contexto escolar, a presença de treinamentos de Autocompaixão constitui um dos eixos centrais, chegando a englobar, em alguns casos, mais de $80 \%$ das atividades e exercícios propostos (Meiklejohn et al., 2012; Zenner et al., 2014). É preciso estar atento, portanto, à estrutura da intervenção, identificando qual o peso atribuído aos exercícios de Mindfulness e àqueles de Autocompaixão (chamados de kindness ou self-compassion exercises). É sabido que cada fator, Mindfulness e Autocompaixão, se comporta de maneira diferente, afetando os desfechos pes- quisados de modo diverso. Igualmente, análises estatísticas de moderação e mediação devem ser conduzidas a fim de compreender melhor o papel de cada fator nos resultados encontrados (Woodruff et al., 2013). Por fim, é necessário verificar a mensuração das variáveis, já que determinadas escalas de Mindfulness avaliam comportamentos autocompassivos; há instrumentos específicos para estes últimos, como a Escala de Auto-compaixão de Neff (2003).

\section{Adaptações Estruturais Necessárias e Protocolos Emergentes}

As primeiras intervenções utilizando Mindfulness no ambiente escolar não seguiam um protocolo padrão, mas se caracterizavam como um conjunto heterogêneo de práticas do tipo mente-corpo, da qual exercícios de Mindfulness faziam parte (Rechtschaffen \& Kabat-Zinn, 2014). A partir de 2008, a fim de organizar uma estrutura homogênea de MBIs para escolas, Thompson e Gauntlett-Gilbert (2008) sugeriram que as adaptações nos protocolos fossem realizadas utilizando modelos de MBIs reconhecidos em sua eficácia, como o MBSR. As principais adaptações deveriam estar relacionadas a: (a) manutenção de estrutura de protocolos; (b) menor duração das práticas; (c) engajamento de pais e cuidadores; (d) maior utilização de práticas denominadas "informais" - inclusão de uma atitude mindful e autocompassiva em atividades cotidianas, ao invés do estabelecimento de um compromisso formal com um exercício específico, por um número exato de minutos por dia; (e) aumento da variedade de práticas (incluindo a incorporação da tecnologia, como a utilização de tablets e internet); (f) utilização de metáforas durante situações de psicoeducação; (g) utilização dos cinco sentidos (maior inclusão de habilidades sensoriomotoras). Zinger (2011) expande este cenário, relatando que um ambiente de sala de aula mindful pode ser verificado a partir da orientação para a execução de tarefas, que devem ser realizadas de modo mais eficaz, com o prevalecimento de pouca perturbação e perspectiva de aceitação e democracia, mesmo diante da emergência de temas polêmicos. 
Desde então, o número de estudos com métodos mais robustos, ou seja, com amostras de tamanho adequado, presença de grupos controle, follow-up (seguimento) e randomização, vêm aumentando (Zenner et al., 2014). As evidências dos efeitos de MBIs para escolas deixaram de ser acumuladas de maneira anedótica, já que os protocolos homogeneizaram as intervenções, estabelecendo exercícios e atividades padronizadas (Zenner et al., 2014). A fim de ilustrar estes avanços, são descritos três protocolos que vêm apresentando resultados promissores.

\section{Dot-be (.B) ou Mindfulness in Schools Program (MiSP)}

O Dot-be ou simplesmente ".B" - um anagrama para dot (pause) e breath (respire) - foi desenvolvido no Reino Unido sobre a estrutura das MBIs protocolares, mais especificamente do $M B C T$. Os autores da intervenção justificam que a manutenção da estrutura protocolar está diretamente associada à tentativa de replicar as evidências positivas presentes nos programas desenvolvidos para a população adulta (Kuyken et al., 2013). Inicialmente, o treinamento foi oferecido para crianças e jovens entre 11 e 18 anos, mas, devido aos resultados iniciais promissores (Kuyken et al., 2013), a faixa etária foi ampliada para crianças a partir dos sete anos de idade. Para esta faixa etária é oferecido o programa chamado Paws-b (Huppert \& Johnson, 2010).

Ainda que a estrutura do Dot-be seja similar àquela das MBIs para adultos, ou seja, seguindo um protocolo, neste as habilidades de Mindfulness são ensinadas através de práticas lúdicas e informais de curta duração (15 minutos). Nas MBIs para adultos existem práticas com até 40 minutos de duração. A estrutura do Dot-be está organizada ao redor de dez temas que podem ser ensinados ao longo de oito a dez encontros. Os temas são: introdução, brincando com a atenção, domando a mente animal, reconhecendo as preocupações, estar aqui e agora, movendo-se no aqui e agora, dar um passo para trás, fazendo amizade com as dificuldades, estar presente com seu coração, e "colocando tudo junto". Cada tema apresenta exercícios de Mindfulness diferentes e complementares, sempre ensinados de forma lúdica. A cada encontro (que pode ser semanal), um tema é incorporado às atividades em sala de aula e no ambiente escolar, e trabalhado durante algum tempo. Através de movimentos corporais (inspirados em artes marciais) e da apresentação de filmes, desenhos e músicas, as habilidades de Mindfulness são transmitidas numa linguagem mais dinâmica e relevante ao contexto infantil e adolescente. Um exemplo é a prática de escaneamento corporal, chamada bed-itation, uma brincadeira com os termos meditation (meditação) e bed (cama). Os momentos de psicoeducação sobre o funcionamento dos processos mentais também são vivenciados de maneira mais informal, com vasta utilização de metáforas (Kuyken et al., 2013). Por fim, no Dot-be há maior ênfase nas práticas e em sua aplicação no mundo, do que na troca de experiências entre participantes (enquiry process), que é bastante valorizada nas MBIs para adultos.

O Dot-be foi desenvolvido para ser incluído diretamente nos currículos (Huppert \& Johnson, 2010); e não como um programa a ser oferecido de modo complementar, fora do horário regular, no ambiente escolar. Assim, o programa deve ser ensinado por um dos membros do Mindfulness in Schools Programs (MiSP) ou por professores treinados diretamente por eles. Até a presente data, mais de duzentos professores foram treinados no programa e mais de trinta escolas ao redor do mundo investigam seus possíveis benefícios (Kuyken et al., 2013).

\section{MindUp}

O MindUp é um protocolo de MBIs desenvolvido para treinar professores e crianças em habilidades de Mindfulness (Roeser et al., 2013; Schonert-Reichl \& Lawlor, 2010). Esta intervenção é composta por práticas Mindfulness adaptadas para execução em sala de aula e tem como objetivos centrais melhorar os níveis de autoconsciência, atenção focada, autorregulação e redução de estresse (Roeser et al., 2013). Outro objetivo exposto pelos autores do programa é o desenvolvimento dos níveis de otimismo e afetos positivos. O MindUp se estrutura através de quatro temas centrais: (a) acalmar a mente (por meio do treino da atenção ao som de um instru- 
mento, um pequeno gongo, ao mesmo tempo em que foca na respiração); (b) atenção Mindful (a uma variedade de sensações, pensamentos e sentimentos); (c) gerenciamento de emoções e de pensamentos negativos; e (d) tomada de consciência de quem eu sou e quem são aqueles que me cercam (Schonert-Reichl \& Lawlor, 2010). É usual a utilização de técnicas de visualização criativa, em conjunto com os exercícios de Mindfulness (Schonert-Reichl \& Lawlor, 2010).

O programa acontece ao longo de dez semanas, período em que as crianças vivenciam uma modificação no currículo, com a inclusão de tarefas desenhadas para fomentar habilidades de Mindfulness na vida cotidiana. $\mathrm{O}$ foco do programa está nas lições diárias, nas quais os estudantes praticam exercícios de autorregulação da atenção, três vezes ao dia, começando com três minutos de cada prática e aumentando este tempo à medida que o programa avança, semana a semana (Schonert-Reichl \& Lawlor, 2010).

No MindUp, como a intenção é a progressiva integração do programa ao currículo regular, os facilitadores da intervenção devem ser os professores em sala de aula, e não profissionais externos formalmente treinados em MBIs para adultos. $\mathrm{O}$ treinamento de educadores no protocolo é uma exigência e acontece ao longo de 12 meses, sendo realizado através de diferentes etapas, presenciais e via internet.

\section{Learning to Breathe (L2B)}

O protocolo Learning to Breath (L2B) "Aprendendo a Respirar" - foi desenvolvido por Broderick e Metz (2009). Trata-se de uma intervenção de Mindfulness para jovens com duração de seis semanas, sendo um encontro por semana. Segundo os autores do $L 2 B$, seu objetivo é o desenvolvimento de autoconsciência corporal e habilidades metacognitivas (Broderick \& Metz, 2009). Assim, um dos propósitos centrais é integrar no dia a dia a habilidade de reduzir autojulgamentos danosos, conduzindo a uma regulação emocional mais eficaz.

A maior parte das adaptações implementadas pelo L2B, em relação aos protocolos para adultos, estão baseadas em releituras dos exercí- cios do MBSR, porém com maior utilização de recursos visuais (fotos e materiais hands-on) e maior incorporação de exercícios baseados em movimentos. Para os alunos mais novos, por exemplo, uma variedade de mini-práticas de Mindfulness são ensinadas para facilitar o início e o encerramento das aulas regulares. Para estes alunos, o ensino acerca das diferentes emoções é realizado através de cartoons (tirinhas) que, segundo os autores, facilitam o reconhecimento de estados emocionais (Brown, Corrigan, \& Higgins-D'Alessandro, 2012). O L2B também ensina como desenvolver "espaços mindful" em sala de aula, através de peace corners (cantos da paz) ou quiet corners (cantos do silêncio) - espaços onde os jovens podem ficar, voluntariamente, por um determinado período de tempo, praticando exercícios de Mindfulness aprendidos no protocolo. O objetivo é a criação in loco de reguladores emocionais que podem ser acionados em situações que antecedem momentos de estresse ou dificuldade, como avaliações ou reuniões (Brown et al., 2012).

\section{Efeitos das Intervenções}

Estudos existentes demonstram que a adaptação das MBIs vem ocorrendo de maneira adequada e com resultados promissores (Frank et al., 2013; Rotne \& Rotne, 2013). Alunos e professores que passam por uma intervenção de Mindfulness adaptada para o ambiente escolar podem desenvolver melhores indicadores gerais de bem estar e autocuidado, performance cognitiva, além de redução de estresse e de sintomas físicos de dor, desconforto e cansaço (Bei et al., 2013; Huppert \& Johnson, 2010; Kuyken et al., 2013; White, 2012; Zenner et al., 2014). As MBIs em escolas podem ser utilizadas também para melhorar indicadores, como a relação estudante/professor, a redução da sintomatologia de Transtorno do Déficit de Atenção e Hiperatividade, a promoção de regulação emocional, e a redução do estresse entre jovens em idade escolar (Frank et al., 2013). Esta série de evidências sugere que os efeitos do treinamento de Mindfulness em ambientes educacionais podem ser 
semelhantes àqueles verificados em adultos em contextos de saúde (Huppert \& Johnson, 2010; Rotne \& Rotne, 2013).

Pesquisas que investigam a acessibilidade $\mathrm{e}$ o impacto do Dot-be nas escolas vêm sendo conduzidas, especialmente com jovens entre 12 e 16 anos (Huppert \& Johnson, 2010; Kuyken et al., 2013). Segundo os autores destes estudos, esta faixa etária é uma janela do desenvolvimento, fundamental para a promoção da autorregulação, um período no qual os estudantes são expostos pela primeira vez a estressores escolares e sociais inerentes a esta etapa do desenvolvimento. Resultados obtidos por Kuyken et al. (2013) sugerem que o treinamento do Dot-be foi bem recebido por professores e estudantes, conseguindo promover bem estar geral e melhores habilidades para lidar com os estressores.

Dois estudos foram conduzidos para verificar os efeitos do MindUp. O primeiro foi realizado com 246 pré-adolescentes norte-americanos, divididos em dois grupos. Metade dos jovens passou por seis programas consecutivos e a outra metade apenas pelas aulas tradicionais (Schonert-Reichl \& Lawlor 2010). Os resultados deste estudo revelaram que os jovens que participaram do MindUp tiveram aumentos significativos nos escores de otimismo e afeto positivo, enquanto aqueles que apenas seguiram o currículo tradicional tiveram decréscimo no otimismo. Também, duas dimensões de competência social e emocional dos professores que passaram pelo treinamento melhoraram: "atenção e concentração" e "competência socioemocional".

O grupo mais jovem de pré-adolescentes que realizaram o programa demonstrou aumentos significativos no autoconceito, em contraste com o grupo controle, para o qual o autoconceito diminuiu. No entanto, o grupo de adolescentes tardios apresentou resultado diferente, sendo que aqueles que passaram pelo treinamento tiveram diminuição no autoconceito, em comparação com o grupo controle, no qual o autoconceito aumentou (Schonert-Reichl \& Lawlor, 2010). Pautados na literatura pesquisada, os autores do estudo sugeriram a existência de um período ótimo para a introdução do treinamento de Mindful- ness, provavelmente anterior à fase adolescente.

Um estudo mais atual (Schonert-Reichl et al., 2015) demonstrou que participantes que receberam a intervenção MIndUP, em comparação com integrantes de grupos de controle, apresentaram melhorias na orientação cognitiva, empatia, capacidade de tomada de perspectiva, regulação emocional, otimismo, autoconceito, maiores diminuições nos sintomas autorrelatados de depressão e agressividade, menor estresse (por medida de cortisolemia), e foram classificados pelos seus pares como mais pró-sociais (por popularidade sociométrica).

Os resultados pós-treino de uma primeira investigação acerca dos efeitos do programa Learning to Breath (L2B) demonstraram que os estudantes que passaram pelo treinamento, quando comparados com um grupo que não recebeu a intervenção, tiveram aumento nas variáveis de calma, relaxamento e Autocompaixão, e redução no número de afetos negativos (Broderick \& Metz, 2009). Outros dados importantes foram a capacidade de melhor regulação emocional e o decréscimo no cansaço e dores (Broderick \& Metz, 2009). Os autores relataram que os adolescentes preferiram realizar atividades e exercícios relevantes para suas demandas corporais, mentais e lúdicas. Assim, atividades de Mindfulness que incorporam tecnologia (smartphones, computadores e outros recursos), esportes, bem como a utilização de mídias diversas - como música e internet constituem outras fontes de motivação importantes, que podem servir como âncoras no desenvolvimento dos exercícios.

Em 2013, a pesquisa foi ampliada, incluindo 216 estudantes de escolas públicas norte-americanas (Metz et al., 2013). Aqueles que participaram da intervenção L2B, em comparação com um grupo controle, que recebeu psicoeducação, apresentaram níveis estatisticamente mais baixos de estresse percebido e de queixas psicossomáticas, além de níveis mais elevados de regulação emocional. $\mathrm{O}$ estudo mais recente envolvendo o L2B foi publicado por Bluth et al. (2015), tendo verificado que a participação no L2B foi mais eficaz na redução de sintomas de- 
pressivos e de estresse em adolescentes do que a participação em um controle (psicoeducação sobre uso de substâncias).

\section{Estratégias de Treinamento Conti- nuado para Professores e Equipe Escolar}

No ambiente escolar, quando um professor, colega de turma, líder estudantil ou membros da administração passam a se comportar de modo mais autorregulado, outros indivíduos tendem a imitar e assimilar esses estados. Este tipo de resposta fica retida e passa a fornecer um modelo para comportamentos futuros (Huppert \& Johnson, 2010). Neste sentido, a modelagem de comportamento se apresenta como uma ferramenta pedagógica que facilita a incorporação (embodiment) das habilidades oriundas do treinamento nas MBIs (Dewar \& Christley, 2013; Germer, 2009). Devido à importância que os modelos exercem no comportamento grupal, o treinamento dos professores e da equipe educacional é fundamental (Meiklejohn et al., 2012). Gestores educacionais, de modo geral, refletem o tom emocional da escola (Rotne \& Rotne, 2013). O treino em Mindfulness ensinado nas MBIs pode trazer benefícios para o indivíduo, mas de forma não apenas circunscrita a ele, já que sua ação no ambiente gera um efeito positivo de "contaminação" (ripple effect; Germer, 2009; Schonert-Reichl \& Lawlor, 2010). Assim, na medida em que comportamentos autorregulados são cultivados de forma deliberada e coletiva, uma ampla gama de recursos de regulação emocional e habilidades pró-sociais pode ser fomentada em todos os níveis da escola (Britton et al., 2014; Rotne \& Rotne, 2013).

As pesquisas realizadas com o Mind $U p$ e L2B indicam que os professores das escolas gostariam de ter mais oportunidades de treinamento em Mindfulness, fato que permitiria explicar com maior facilidade aos pais o objetivo do treinamento que é realizado com as crianças. A meta-análise conduzida por Zenner et al. (2014) evidenciou que o treinamento de professores é o grande desafio a ser superado para o avanço metodológico e maior efetividade das MBIs em escolas.
Os primeiros treinamentos realizados com professores usaram modelos de cursos intensivos de curta duração, que ensinam como utilizar as técnicas em sala de aula. Apesar dos professores aprenderem os exercícios de maneira eficaz, quando emerge uma questão mais complexa sobre o funcionamento cognitivo ou alguma dificuldade em se regular as emoções, estes ficam inseguros (Schonert-Reichl \& Lawlor, 2010). Conduzir um exercício ou explicar a teoria de Mindfulness é relativamente simples, mas as verdadeiras habilidades são expostas quando o professor de Mindfulness se depara com dúvidas e comentários que surgem da idiossincrasia de cada participante (Burke, 2010).

$\mathrm{O}$ problema que orbita o treinamento e suporte oferecido para os professores e gestores da educação é crítico (Crane \& Kuyken, 2013; Crane et al., 2012; Meiklejohn et al., 2012; Napoli, Krech, \& Holley, 2005; Rotne \& Rotne, 2013). A influência da experiência individual dos facilitadores de Mindfulness é tema recorrente na literatura, e não pode ser ignorada (Crane \& Kuyken, 2013). No contexto da saúde mental, pacientes avaliados por terapeutas que praticaram Mindfulness apresentaram menos sintomas negativos de saúde, quando comparados com pacientes atendidos por terapeutas que nunca praticaram (Grepmair et al., 2007). Estas evidências remetem à importância do envolvimento, em primeiro lugar, com a prática individual. Um formato de treinamento breve, na forma de cursos intensivos, parece ser insuficiente para fomentar em professores a expertise necessária para transmitir as habilidades de Mindfulness em sala de aula. Para que isso aconteça, é fundamental o aprofundamento na prática pessoal (Carmody \& Baer, 2008; Crane et al., 2013). Neste sentido, o Dot-be se difere dos demais protocolos, ao exigir experiência prévia e certificação formal do profissional interessado em se tornar um instrutor de MBIs nas escolas. Este parece ser um ponto central, pois é sabido que os anos de experiência dos facilitadores, bem como suas habilidades gerais em transmitir os conhecimentos, afetam diretamente os resultados esperados (Carmody $\&$ Baer, 2008; Khoury et al., 2013). No entanto, não são os professores da escola que recebem 
o treinamento e instruem os alunos em sala de aula (Huppert \& Johnson, 2010). Portanto, se o problema do tempo de prática dos instrutores é parcialmente solucionado, permanece a falta de familiaridade destes profissionais com a realidade cotidiana dos alunos, professores, familiares e ambiente escolar. É bastante provável que os treinamentos de formação em Mindfulness para professores se expandam para um modelo híbrido, que não apenas priorize a prática e experiência pessoal, mas inclua os profissionais habituados com as idiossincrasias de seus alunos e ambientes escolares (Zenner et al., 2014).

Nos Estados Unidos, um programa de oito semanas foi elaborado para apoiar os professores neste processo (Roeser et al., 2013). Este programa vem sendo desenvolvido no MindUp através de uma variedade de exercícios e atividades pedagógicas que promovem Mindfulness, Autocompaixão e estratégias de resiliência emocional entre professores e gestores educacionais. Cinco atividades fundamentais de ensino sobre Mindfulness e Autocompaixão são ministradas aos professores: (a) práticas orientadas de Mindfulness e ioga; (b) discussão em grupo sobre a prática; (c) atividades em pequenos grupos, para a prática das habilidades na vida real; (d) aulas e atribuição de trabalhos dirigidos; e (e) tarefas metacognitivas e comportamentais para serem realizadas em casa, no dia a dia (Roeser et al., 2013). Outras técnicas são exploradas durante o processo, tais como o desenvolvimento de um diário mindful e a criação de palestras didáticas. Os resultados deste estudo demonstram que $87 \%$ dos professores que completam o programa relatam o benefício do treinamento em suas vidas (Roeser et al., 2013). Professores que participaram deste grupo experimental demonstraram, ao final do programa, maiores níveis de Mindfulness, atenção focada, Autocompaixão, utilização mais eficiente da memória de trabalho, baixos níveis de estresse ocupacional, bem como ausência de sinais de burnout. Em um seguimento (follow-up) de três meses, quando foram comparados com um grupo controle, os resultados obtidos permaneceram. Apesar dos evidentes benefícios deste treinamento, os dados demonstrando como a participação neste programa está influenciando as habilidades dos professores em manejar e fomentar estratégias de Mindfulness e Autocompaixão nas salas de aula ainda não foram publicados.

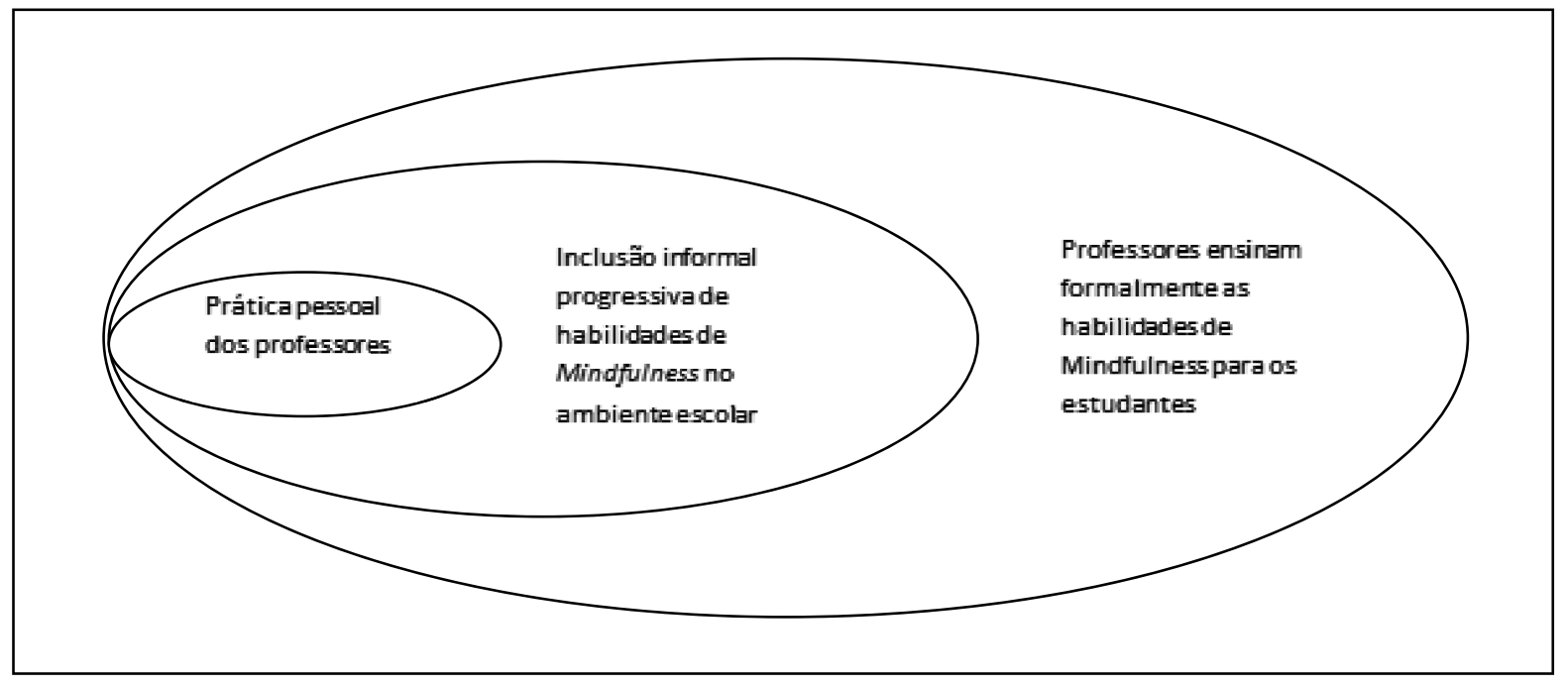

Figura 1. Diferentes níveis de incorporação de habilidades de Mindfulness no sistema educacional.

A Figura 1 esboça a estratégia utilizada para facilitar a transmissão das habilidades de Mindfulness no contexto escolar. Na etapa da elipse menor, os professores e gestores educacionais recebem o treinamento em Mindfulness, inician- do sua prática e desenvolvimento pessoal. Este primeiro passo é fundamental, posto que, para os professores ensinarem aos alunos questões sobre atenção, autocuidado e gentileza consigo, os alunos precisam observar ações efetivas que de- 
notem regulação emocional, gerenciamento do estresse e Autocompaixão. Na segunda elipse, sugere-se um engajamento progressivo de professores no desenvolvimento de ações estratégicas e pontuais de incorporação de Mindfulness nas salas de aula. Estas ações podem ser efetivadas incluindo-se pequenos exercícios antes e depois do início das atividades diárias. Finalmente, na terceira e maior elipse, sugere-se a transmissão formal das habilidades através, por exemplo, da implementação do modelo de encontros semanais e acompanhamento semanal, como nas MBIs. A ideia é promover Mindfulness de modo gradual e progressivo nos ambientes escolares (Coles, 2015).

\section{Considerações Finais}

Embora as intervenções apresentadas sejam promissoras e procurem superar as limitações apontadas por Britton et al. (2014) e Burke (2010), estes modelos ainda necessitam ser melhor testados em diferentes amostras. Muitas MBIs para escolas têm surgido e o contínuo desenvolvimento de novos protocolos é uma realidade. É fundamental, no entanto, a replicação e o aperfeiçoamento dos protocolos já existentes, para que não ocorra unicamente um acúmulo de evidências isoladas.

Uma maior sensibilização da população acadêmica sobre o assunto, especialmente de pesquisadores interessados em temas como o bem estar e desempenho de crianças e jovens em ambientes escolares, pode contribuir para a expansão das pesquisas. Além disso, a apropriação por parte de gestores educacionais e professores, ou seja, aqueles que vivenciam a realidade prática deste universo, pode contribuir com esse processo. Do mesmo modo, um contato mais ampliado entre pesquisadores e educadores é capaz de potencializar a implementação das MBIs em escolas. A partir do sucesso destas propostas, seria viável a inclusão progressiva de um processo de treinamento da equipe educacional em habilidades de Mindfulness. As evidências demonstram que tal processo deve ser conduzido seguindo as evidências de pesquisa que indicam quais modelos e ferramentas são mais efetivas para esse fim. Progressivamente, pode-se viabilizar o desenho de diretrizes básicas e protocolos padronizados que funcionem de acordo com as demandas específicas de cada ambiente escolar.

No Brasil, iniciativas pioneiras estão surgindo, como o projeto "Meditação pela Paz". Apesar do projeto não configurar uma Intervenção Baseada em Mindfulness, propriamente, inclui em seu protocolo exercícios breves de atenção na respiração. O cerne da prática, no entanto, está no desenvolvimento de estados compassivos que emergem de exercícios do tipo "mantra". Resultados preliminares, ainda não publicados, de uma pesquisa realizada por membros do Grupo de Pesquisa em Neuropsicologia Clínica e Experimental (GNCE) da Pontifícia Universidade Católica do Rio Grande do Sul (PUCRS), verificou os efeitos deste treinamento em alunos de uma escola pública de Porto Alegre com idades entre 10 e 14 anos. As análises demonstraram que a intervenção promoveu melhoras em componentes de velocidade de processamento e no acesso a informações implícitas (processamento pragmático-inferencial e de teoria da mente). Além disso, incrementou componentes de inibição e iniciação verbal das funções executivas. Outro programa brasileiro em avaliação foi estruturado ao longo de doze semanas, combinando Mindfulness e estratégias de aprendizagem socioemocional (M-SEL). A pesquisa, também conduzida em Porto Alegre, demonstrou que alunos do quinto ano do ensino fundamental que receberam a intervenção apresentaram melhorias significativas em escores de saúde mental e qualidade de vida (Ovídio et al., 2016). Este cenário mostra que grupos brasileiros têm procurado avançar para além de estudos teóricos e revisões sobre Mindfulness, iniciando a investigação empírica sobre os efeitos de MBIs em populações jovens em ambientes escolares.

\section{Referências}

Barros, V. V. de, Kozasa, E. H., Souza, I. C. W. de, \& Ronzani, T. M. (2014). Validity evidence of the Brazilian version of the Five Facet Mindfulness Questionnaire (FFMQ). Psicologia: Teoria e Pesquisa, 30(3), 317-327. doi:10.1590/S010237722014000300009 
Bei, B., Byrne, M. L., Ivens, C., Waloszek, J., Woods, M. J., Dudgeon, P., ...Allen, N. B. (2013). Pilot study of a mindfulness-based, multi-component, in-school group sleep intervention in adolescent girls. Early Intervention in Psychiatry, 7(2), 213220. doi:10.1111/j.1751-7893.2012.00382.x

Bluth, K., Campo, R. A., Pruteanu-Malinici, S., Reams, A., Mullarkey, M., \& Broderick, P. C. (2015). A school-based mindfulness pilot study for ethnically diverse at-risk adolescents. Mindfulness, 1-15. doi:10.1007/s12671-0140376-1

Britton, W. B., Lepp, N. E., Niles, H. F., Rocha, T., Fisher, N. E., \& Gold, J. S. (2014). A randomized controlled pilot trial of classroom-based mindfulness meditation compared to an active control condition in sixth-grade children. Journal of School Psychology, 52(3), 263-278. doi:10.1016/j.jsp.2014.03.002

Broderick, P. C., \& Metz, S. (2009). Learning to BREATHE: A pilot trial of a mindfulness curriculum for adolescents. Advances in School Mental Health Promotion, 2(1), 35-46. doi:10.1 080/1754730X.2009.9715696

Brown, K. W., \& Ryan, R. M. (2006). Perils and promise in defining and measuring mindfulness: Observations from experience. Clinical Psychology: Science and Practice, 11(3), 242-248. doi:10.1093/clipsy.bph078

Brown, P. M., Corrigan, M. W., \& Higgins-D’Alessandro, A. (Eds.). (2012). Handbook of prosocial education. Lanham, MD: Rowman \& Littlefield.

Burke, C. A. (2010). Mindfulness-based approaches with children and adolescents: A preliminary review of current research in an emergent field. Journal of Child and Family Studies, 19(2), 133144. doi:10.1007/s10826-009-9282-x

Carmody, J., \& Baer, R. A. (2008). Relationships between mindfulness practice and levels of mindfulness, medical and psychological symptoms and well-being in a mindfulnessbased stress reduction program. Journal of Behavioral Medicine, 31(1), 23-33. doi:10.1007/ s10865-007-9130-7

Chiesa, A., \& Serretti, A. (2009). Mindfulness-based stress reduction for stress management in healthy people: A review and meta-analysis. Journal of Alternative and Complementary Medicine, 15(5), 593-600. doi:10.1089/acm.2008.0495
Coles, M. I. (Ed.). (2015). Towards the compassionate school from golden rule to golden thread. London: Trentham Books.

Crane, R. S., Eames, C., Kuyken, W., Hastings, R. P., Williams, J. M. G., Bartley, T., ...Surawy, C. (2013). Development and validation of the mindfulness-based interventions - teaching assessment criteria (MBI:TAC). Assessment, 20(6), 681-688. doi:10.1177/1073191113490790

Crane, R. S., \& Kuyken, W. (2013). The implementation of mindfulness-based cognitive therapy: Learning from the UK health service experience. Mindfulness, 4, 246-254. doi:10.1007/s12671012-0121-6

Crane, R. S., Kuyken, W., Williams, J. M. G., Hastings, R. P., Cooper, L., \& Fennell, M. J. V. (2012). Competence in teaching mindfulness-based courses: Concepts, development and assessment. Mindfulness, 3(1), 76-84. doi:10.1007/ s12671-011-0073-2

Cullen, M. (2011). Mindfulness-based interventions: An emerging phenomenon. Mindfulness, 2(3), 186-193. doi:10.1007/s12671-011-0058-1

Dewar, B., \& Christley, Y. (2013). A critical analysis of compassion in practice. Nursing Standard, 28(10), 46-50. doi:10.7748/ns2013.11.28.10.46. e7828

Duarte, C., Ferreira, C., \& Gouveia, J. P. (2010). O traço de Mindfulness como protector nos comportamentos bulímicos. Psychologica, 52(2), 401-422.

Eberth, J., \& Sedlmeier, P. (2012). The effects of mindfulness meditation: A meta-analysis. Mindfulness, 3(3), 174-189. doi:10.1007/s12671 -012-0101-x

Frank, J. L., Jennings, P. A., \& Greenberg, M. T. (2013). Mindfulness-based interventions in school settings: An introduction to the special issue. Research in Human Development, 10(3), 205-210. doi:10.1080/15427609.2013.818480

Germer, C. K. (2009). The mindful path to selfcompassion: Freeing yourself from destructive thoughts and emotions. New York: Guilford Press.

Grepmair, L., Mitterlehner, F., Loew, T., Bachler, E., Rother, W., \& Nickel, M. (2007). Promoting mindfulness in psychotherapists in training influences the treatment results of their patients: A randomized, double-blind, controlled study. 
Psychotherapy and Psychosomatics, 76(6), 332338. doi:10.1159/000107560

Grossman, P., Niemann, L., Schmidt, S., \& Walach, H. (2004). Mindfulness-based stress reduction and health benefits. A meta-analysis. Journal of Psychosomatic Research, 57(1), 35-43. doi:10.1016/S0022-3999(03)00573-7

Hayes, A. M., \& Feldman, G. (2004). Clarifying the construct of mindfulness in the context of emotion regulation and the process of change in therapy. Clinical Psychology: Science and Practice, 11(3), 255-262. doi:10.1093/clipsy.bph080

Hayes, S. C., Follette, V. M., \& Linehan, M. M. (Eds.). (2011). Mindfulness and Acceptance: Expanding the cognitive-behavioral tradition. New York: The Guilford Press.

Hirayama, M. S., Milani, D., Rodrigues, R. C. M., Barros, N. F. de, Alexandre, N. M. C., Hirayama, M. S., ...Alexandre, N. M. C. (2014). The perception of behavior related to mindfulness and the Brazilian version of the Freiburg Mindfulness Inventory. Ciência \& Saúde Coletiva, 19(9), 3899-3914. doi:10.1590/141381232014199.12272013

Hölzel, B. K., Lazar, S. W., Gard, T., Schuman-Olivier, Z., Vago, D. R., \& Ott, U. (2011). How does mindfulness meditation work? Proposing mechanisms of action from a conceptual and neural perspective. Perspectives on Psychological Science, 6(6), 537-559. doi:10.1177/1745691611419671

Huppert, F. A., \& Johnson, D. M. (2010). A controlled trial of mindfulness training in schools: The importance of practice for an impact on well-being. The Journal of Positive Psychology, 5(4), 264 274. doi:10.1080/17439761003794148

Joyce, A., Etty-Leal, J., Zazryn, T., \& Hamilton, A. (2010). Exploring a Mindfulness Meditation Program on the Mental Health of Upper Primary Children: A pilot study. Advances in School Mental Health Promotion, 3(2), 17-25. doi:10.1 080/1754730X.2010.9715677

Kabat-Zinn, J. (1982). An outpatient program in behavioral medicine for chronic pain patients based on the practice of mindfulness meditation: Theoretical considerations and preliminary results. General Hospital Psychiatry, 4(1), 33-47.

Kabat-Zinn, J. (2003). Mindfulness-based interventions in context: Past, present, and future. Cli- nical Psychology: Science and Practice, 10(2), 144-156. doi:10.1093/clipsy.bpg016

Khoury, B., Lecomte, T., Fortin, G., Masse, M., Therien, P., Bouchard, V., ...Hofmann, S. G. (2013). Mindfulness-based therapy: A comprehensive meta-analysis. Clinical Psychology Review, 33(6), 763-771. doi:10.1016/j.cpr.2013.05.005

Kuyken, W., Weare, K., Ukoumunne, O. C., Vicary, R., Motton, N., Burnett, R., ...Huppert, F. (2013). Effectiveness of the mindfulness in schools programme: Non-randomised controlled feasibility study. The British Journal of Psychiatry: The Journal of Mental Science, 203(2), 126131. doi:10.1192/bjp.bp.113.126649

Langer, E. J. (1992). Matters of mind: Mindfulness/ mindlessness in perspective. Consciousness and Cognition, 1(3), 289-305. doi:10.1016/1053-8100(92)90066-J

Ludwig, D. S., \& Kabat-Zinn, J. (2008). Mindfulness in medicine. JAMA: The Journal of the American Medical Association, 300(11), 1350-1352. doi:10.1001/jama.300.11.1350

Meiklejohn, J., Phillips, C., Freedman, M. L., Griffin, M. L., Biegel, G., Roach, A., ...Saltzman, A. (2012). Integrating mindfulness training into K-12 Education: Fostering the resilience of teachers and students. Mindfulness, 3(4), 291-307. doi:10.1007/s12671-012-0094-5

Menezes, C. B., Dell'Aglio, D. D., \& Bizarro, L. (2012). Meditação, bem-estar e a ciência psicológica: Revisão de estudos empíricos. Interação em Psicologia, 15(2). Recuperado em http://ojs. c3sl.ufpr.br/ojs2/index.php/psicologia/article/ view/20249

Metz, S., Frank, J., Reibel, D., Cantrell, T., Sanders, R., \& Broderick, P. (2013). The Effectiveness of the Learning to BREATHE Program on Adolescent Emotion Regulation. Jefferson Myrna Brind Center of Integration Medicine Faculty Papers. Retrieved from http://jdc.jefferson.edu/ jmbcimfp/15

Napoli, M., Krech, P. R., \& Holley, L. C. (2005). Mindfulness training for elementary school students. Journal of Applied School Psychology, 21(1), 99-125. doi:10.1300/J370v21n01_05

Neff, K. D. (2003, July-September). The development and validation of a Scale to Measure Self-Compassion. Self and Identity, 223-250. doi:10.1080/15298860309027 
Neff, K. D., \& Germer, C. K. (2013). A pilot study and randomized controlled trial of the Mindful Self-Compassion Program. Journal of Clinical Psychology, 69(1), 28-44. doi:10.1002/ jclp.21923

Neff, K. D., Rude, S. S., \& Kirkpatrick, K. L. (2007). An examination of self-compassion in relation to positive psychological functioning and personality traits. Journal of Research in Personality, 4l(4), 908-916. doi:10.1016/j.jrp.2006.08.002

Ovídio, W. J., Rigatti, R., Menezes, C. B., Guimarães, G., Faceto, O., \& Heldt, E. (2016). Impact of a combined mindfulness and social-emotional learning program on fifth graders in a Brazilian public school setting. Psychology \& Neuroscience, 9(1), 79-90.

Rechtschaffen, D., \& Kabat-Zinn, J. (2014). The way of mindful education: Cultivating well-being in teachers and students. New York: W. W. Norton \& Company.

Roeser, R. W., Schonert-Reichl, K. A., Jha, A., Cullen, M., Wallace, L., Wilensky, R., ...Harrison, J. (2013). Mindfulness training and reductions in teacher stress and burnout: Results from two randomized, waitlist-control field trials. Journal of Educational Psychology, 105(3), 787-804. doi:10.1037/a0032093

Rotne, K., \& Rotne, D. F. (2013). Everybody present: Mindfulness in education. Berkeley, CA: Parallax Press.

Russell, T. A., \& Tatton-Ramos, T. P. (2015). Compassionate minds and brains in schools. In M. I. Coles (Ed.), Towards the compassionate school from golden rule to golden thread. London, UK: Trentham Books.

Schonert-Reichl, K. A., \& Lawlor, M. S. (2010). The effects of a mindfulness-based education program on pre- and early adolescents' well-being and social and emotional competence. Mindfulness, 1(3), 137-151. doi:10.1007/s12671-0100011-8

Schonert-Reichl, K. A., Oberle, E., Lawlor, M. S., Abbott, D., Thomson, K., Oberlander, T. F., \& Diamond, A. (2015). Enhancing cognitive and social-emotional development through a simple-to-administer mindfulness-based school program for elementary school children: A randomized controlled trial. Developmental Psychology, 51(1), 52-66. doi:10.1037/a0038454
Silveira, A. da C. da, Castro, T. G. de, \& Gomes, W. B. (2012). Adaptation and validation of the Philadelphia Mindfulness Scale among Brazilian adults. Psico-USF, 17(2), 215-223. doi:10.1590/ S1413-82712012000200005

Tang, Y.-Y., \& Posner, M. I. (2009). Attention training and attention state training. Trends in $\mathrm{Cog}$ nitive Sciences, 13(5), 222-227. doi:10.1016/j. tics.2009.01.009

Tatton-Ramos, T. P. (2015). O que é Mindfulness? In P. Lucena-Santos, J. Pinto-Gouveia, \& M. da S. Oliveira (2015), Terapias comportamentais de terceira geração: Um guia para profissionais (pp. 59-80). Novo Hamburgo, RS: Sinopsys.

Teasdale, J. D., Segal, Z. V., Williams, J. M. G., Ridgeway, V. A., Soulsby, J. M., \& Lau, M. A. (2000). Prevention of relapse/recurrence in major depression by mindfulness-based cognitive therapy. Journal of Consulting and Clinical Psychology, 68(4), 615-623. doi:10.1037//0022-006X.68.4.615

Thompson, M., \& Gauntlett-Gilbert, J. (2008). Mindfulness with children and adolescents: effective clinical application. Clinical Child Psychology and Psychiatry, 13(3), 395-407. doi:10.1177/1359104508090603

Vandenberghe, L., \& Sousa, A. C. A. de. (2006). Mindfulness nas terapias cognitivas e comportamentais. Revista Brasileira de Terapias Cognitivas, 2(1), 35-44.

Walsh, J. J., Balint, M. G., Smolira, D. R., Fredericksen, L. K., \& Madsen, S. (2009). Predicting individual differences in mindfulness: The role of trait anxiety, attachment anxiety and attentional control. Personality and Individual Differences, 46(2), 94-99. doi:10.1016/j. paid.2008.09.008

White, L. S. (2012). Reducing stress in school-age girls through mindful yoga. Journal of Pediatric Health Care: Official Publication of National Association of Pediatric Nurse Associates \& Practitioners, 26(1), 45-56. doi:10.1016/j.pedhc.2011.01.002

Williams, J. M. G., \& Kuyken, W. (2012). Mindfulness-based cognitive therapy: A promising new approach to preventing depressive relapse. The British Journal of Psychiatry: The Journal of Mental Science, 200(5), 359-360. doi:10.1192/ bjp.bp.111.104745 
Woodruff, S. C., Glass, C. R., Arnkoff, D. B., Crowley, K. J., Hindman, R. K., \& Hirschhorn, E. W. (2013). Comparing self-compassion, mindfulness, and psychological inflexibility as predictors of psychological health. Mindfulness, 1-12. doi:10.1007/s12671-013-0195-9

Zenner, C., Herrnleben-Kurz, S., \& Walach, H. (2014). Mindfulness-based interventions in schools: A systematic review and meta-analysis. Frontiers in Psychology, 5. doi:10.3389/fpsyg.2014.00603
Zinger, L. (2011). Educating for tolerance and compassion: Is there a place for meditation in a college classroom? College Teaching Methods \& Styles Journal (CTMS), 4(4), 25-28.

Recebido: $1 \% 04 / 2015$

$1^{a}$ revisão: $15 / 11 / 2015$

Aceite final: 19/11/2015 